\title{
Correction: Author's Responses to Peer Reviews of "Forecasting the COVID-19 Pandemic in Saudi Arabia Using a Modified Singular Spectrum Analysis Approach: Model Development and Data Analysis"
}

Nader Alharbi, $\mathrm{PhD}$

King Saud bin Abdulaziz University for Health Sciences, King Abdullah International Medical Research Center, Riyadh, Saudi Arabia

\section{Corresponding Author:}

Nader Alharbi, $\mathrm{PhD}$

King Saud bin Abdulaziz University for Health Sciences

King Abdullah International Medical Research Center

Prince Mutib Ibn Abdullah Ibn Abdulaziz Rd

Ar Rimayah

Riyadh,

Saudi Arabia

Phone: 966114299999 ext 95590

Email: alharbina@ksau-hs.edu.sa

\section{Related Article:}

Correction of: https://xmed.jmir.org/2021/1/e28742

(JMIRx Med 2021;2(2):e29879) doi: 10.2196/29879

In "Author's Responses to Peer Reviews of 'Forecasting the COVID-19 Pandemic in Saudi Arabia Using a Modified Singular Spectrum Analysis Approach: Model Development and Data Analysis"” (JMIRx Med 2021;2(1):e28742) one error was noted.

The reviewer IDs of two anonymous reviewers have been removed from the published article to preserve anonymity.
The correction will appear in the online version of the paper on the JMIR Publications website on April 26, 2021, together with the publication of this correction notice. Because this was made after submission to PubMed, PubMed Central, and other full-text repositories, the corrected article has also been resubmitted to those repositories.

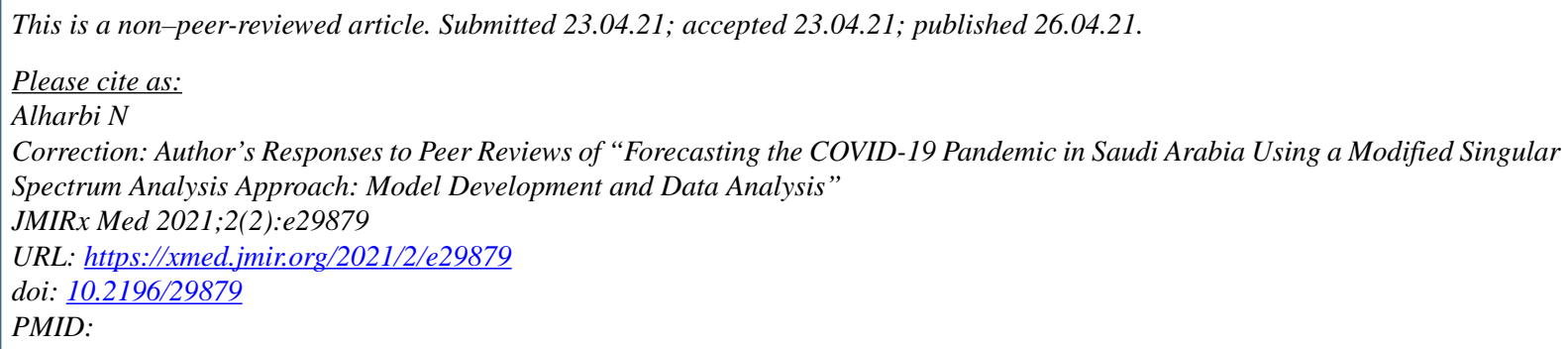

CNader Alharbi. Originally published in JMIRx Med (https://xmed.jmir.org), 26.04.2021. This is an open-access article distributed under the terms of the Creative Commons Attribution License (https://creativecommons.org/licenses/by/4.0/), which permits unrestricted use, distribution, and reproduction in any medium, provided the original work, first published in JMIRx Med, is properly cited. The complete bibliographic information, a link to the original publication on https://xmed.jmir.org/, as well as this copyright and license information must be included. 